\title{
System Level Challenges and Resolutions in Organ Donation and Transplantation Program in Qatar
}

Hassan Al-Thani ${ }^{1}$, Tulika Mehta Agarwal ${ }^{2}$, Rajvir Singh $^{3^{*}}$, Betsy Varughese ${ }^{2}$, Yousuf Al-Maslamani ${ }^{4}$ and Ayman El-Menyar $^{1}$

${ }^{1}$ Department of Trauma and Surgery, Hamad Medical Corporation, Qatar

2Organ Donation Project, QNRF Hamad Medical Corporation, Qatar

${ }^{3}$ Department of Cardiology, Hamad Medical Corporation, Qatar

${ }^{4}$ Department of Head Transplantation, Hamad Medical Corporation, Qatar

*Corresponding author: Rajvir Singh, Senior Consultant, Department of Cardiology, Hamad Medical Corporation, Qatar, Tel: +974-55897044/44390442; E-mail: rajvir.aiims@gmail.com

Received date: February 20, 2018; Accepted date: February 26, 2018; Published date: March 05, 2018

Copyright: (c) 2018 Al-Thani $\mathrm{H}$, et al. This is an open-access article distributed under the terms of the Creative Commons Attribution License, which permits unrestricted use, distribution and reproduction in any medium, provided the original author and source are credited.

\begin{abstract}
Introduction: Organ donation and transplantation programs are affected by factors impacting donation decisions of individuals. These factors have been widely researched world over. However, few researches have studied the organ donation system level challenges that jeopardize the system even when prospective donors are available. It is important to understand these challenges and find appropriate resolutions for them. This qualitative study was conducted to understand the challenges and resolutions in the organ donation and transplant system of Qatar.
\end{abstract}

Methodology: Eight focus group discussions were carried out with homogenous groups of health care workers working in the field of organ donation or organ transplantation in Qatar. After consenting, they were asked open ended questions by an unbiased moderator. Each session lasted for 60-90 min and ended with session summary and participant feedback. The question workbooks were prepared after extensive review of literature and discussion with non-participating stake holders of the organ donation and transplant program in Qatar. Discussions were audio recorded, transcribed and later coded and analyzed on Nvivo software. COREQ checklist was used while reporting the results of this study.

Results: Underutilization of potential donors, organ donation campaign, brain dead donors, family consenting, living donation, infrastructure, staff training, allocation and recruitment were main system level challenges to resolve to ensure maximum utilization of potential donors and donated organs.

Conclusion: Inclusion of multidisciplinary teams during organ transplant, increased representation from departments doing transplants in transplant committee, minimizing wastage of available organs, streamlining the process of brain dead identification, declaration and maintenance of deceased organ, centralized facility for living donor work up and improved infrastructural facility were advocated to improve utilization of donated organs to resolve system level challenges. Improvement in after care and compliance in transplanted patients, registering higher number of donors and increasing family consent by ensuring communication of donation decision to family are the need of the hour.

Keywords: System issues; Challenges in organ donation; Transplantation challenges; Phenomenological study; Focus group discussions; Organ donation

\section{Introduction}

Organ donation and transplantation have progressed world over due to improved awareness and availability, better surgical methods, preservation of organ, and advances in immune-suppressive therapies. Organ transplant has become a critical management therapy for severe organ failure. Living and deceased organ donations are the two types of organ donations that are used for procuring organs from individuals. Deceased donation which takes place after the death of the donor, has been found to be highest in opt out countries such as Spain and Sweden besides others [1], but Qatar being an opt-in country, the major source of organs for transplant come from living donors and deceased donors after brain death contribute in relatively much lower numbers to the available organ pool [2]. Non-heart-beating donation is still not available in the country. There is an increasing demand for transplantable kidneys and livers due to increasing trend of end stage renal and liver diseases world over and in Qatar and other GCC countries (Global Cooperation Council) [3,4]. Qatar is faced with several challenges at the system level that hinder the organ donation and transplantation program. Healthcare personnel are a key factor for the success or failure of transplant programs [5]. Their perception and opinions on the donation process, for example, registration of potential donors, are highly influential in the way people think about the process [6-8].

In the present study, various system level challenges faced by the organ donation and transplant teams were brought out by health care workers working in the field of organ donation and transplantation in Qatar. A qualitative method using focus group discussions was used in the present study so that an open and non-threatening forum could be 
Citation: Al-Thani H, Agarwal TM, Singh R, Varughese B, Al-Maslaman Y, et al. (2018) System Level Challenges and Resolutions in Organ

Page 2 of 13

provided to health care workers for carrying out discussions about system level challenges in organ donation and transplant program of Qatar as it is difficult to capture expressive information in quantitative data form [9]. Their proposed recommendations might be useful to resolve the prevailing system level issues.

\section{Methodology}

Eight focus group discussions were conducted with 40 health care workers working in organ donation or transplantation process in the Government Health Organization of Qatar. The participants were representative of their work specialist and included males and females belonging to Arabic or non-Arabic ethnicities.

Interview guides were prepared using open-ended questions by the research team. Audio recordings were carried out during the session for ease and accuracy of data transcription. The focus group discussions were organized between March-April 2015 in a neutral venue at the Medical Research Center. Each session lasted for duration of 60-90 minutes. Research assistants and transcribers were present with the moderator for taking field notes of the discussions.

Participants were provided with copies as well as read out verbal informed consent to participate in the study before starting the discussion. This also included information about the study, audio recording of data, data confidentiality and security related details and how data would be used. After listening to the information participants provided verbal consent and indicated agreement to participate by continuing to be a part of the discussion. Every focus group discussion ended with communication of summary of the session to the participants and their feedback.
The data was coded using NVivo software and was used to categorize the system level issues and to analyze the interrelationship between different codes. Illustrative quotations were presented for each theme identified along with the participant code number.

\section{Theory and methodological orientation}

The present study was grounded in the Theory of Planned behavior by Ajzen Icek, 1991 [10]. This theory is used to predict the behavior of individual and explains why an individual chose to indulge in or refrain from a particular behavior. Focused group discussions were conducted to generate data from the health care workers who were involved in different roles in the organ donation and transplant process. The main themes were identified before the coding of the data. However, content of the data obtained after the coding was analyzed for the sub themes that emerged. The results of the present study have been reported based on COREQ guidelines for reporting qualitative studies [11] Detailed description of study methodology is described [12].

\section{Results}

The main themes that were identified during the focus group discussions were organ donation campaign, brain dead donor related challenge, underutilization of available organs, challenges in getting family consent, changing priorities of administration, living donor work-up, infrastructural scarcity in hospital and staff challenges. The conceptual links relationships among themes and subthemes are portrayed in Table 1. Illustrative quotations for each theme are provided in Table 2.

\begin{tabular}{|c|c|c|c|c|c|c|}
\hline \multirow{2}{*}{$\begin{array}{l}\text { Main Themes } \\
\begin{array}{l}\text { Organ donation } \\
\text { campaign challenges }\end{array}\end{array}$} & \multicolumn{6}{|l|}{ Sub Themes } \\
\hline & $\begin{array}{l}\text { Infrastructural } \\
\text { scarcity }\end{array}$ & Staff training & Lure of incentives & $\begin{array}{l}\text { Communication } \\
\text { donation decision }\end{array}$ & - & - \\
\hline $\begin{array}{l}\text { Brain dead donor } \\
\text { challenges }\end{array}$ & $\begin{array}{l}\text { Brain dead } \\
\text { declaration }\end{array}$ & Donor maintenance & - & - & - & - \\
\hline Underutilization & $\begin{array}{l}\text { Lack of supporting } \\
\text { law }\end{array}$ & High organ rejection & Communication gap & $\begin{array}{l}\text { Absence of non- heart } \\
\text { beating donation }\end{array}$ & $\begin{array}{l}\text { Lack of policy } \\
\text { compliance }\end{array}$ & $\begin{array}{l}\text { Existing law and } \\
\text { protocols do not } \\
\text { support organ } \\
\text { donation }\end{array}$ \\
\hline $\begin{array}{l}\text { Family consent } \\
\text { challenges }\end{array}$ & Language barrier & $\begin{array}{l}\text { Lack of face to face } \\
\text { communication }\end{array}$ & - & - & - & - \\
\hline Living donor work up & Protocol lacking & $\begin{array}{l}\text { Lack of centralized } \\
\text { facility }\end{array}$ & - & - & - & - \\
\hline Infrastructural scarcity & $\begin{array}{l}\text { In organ donation } \\
\text { campaigns }\end{array}$ & In hospitals facility & - & - & - & - \\
\hline $\begin{array}{l}\text { Changing priorities of } \\
\text { administration }\end{array}$ & - & - & - & - & - & - \\
\hline Staff challenges & Staff scarcity & Staff training & Staff allocation & - & - & - \\
\hline
\end{tabular}

Table 1: Main themes and sub themes that emerged from the discussions.

\begin{tabular}{|l|l|}
\hline Challenge & Illustrative Statement \\
\hline Campaign challenges & SP1/TE4: More training is required here in Qatar especially for the volunteers. \\
\hline
\end{tabular}


Citation: Al-Thani H, Agarwal TM, Singh R, Varughese B, Al-Maslaman Y, et al. (2018) System Level Challenges and Resolutions in Organ Donation and Transplantation Program in Qatar. J Transplant Technol Res 8: 178. doi:10.4172/2161-0991.1000178

Page 3 of 13

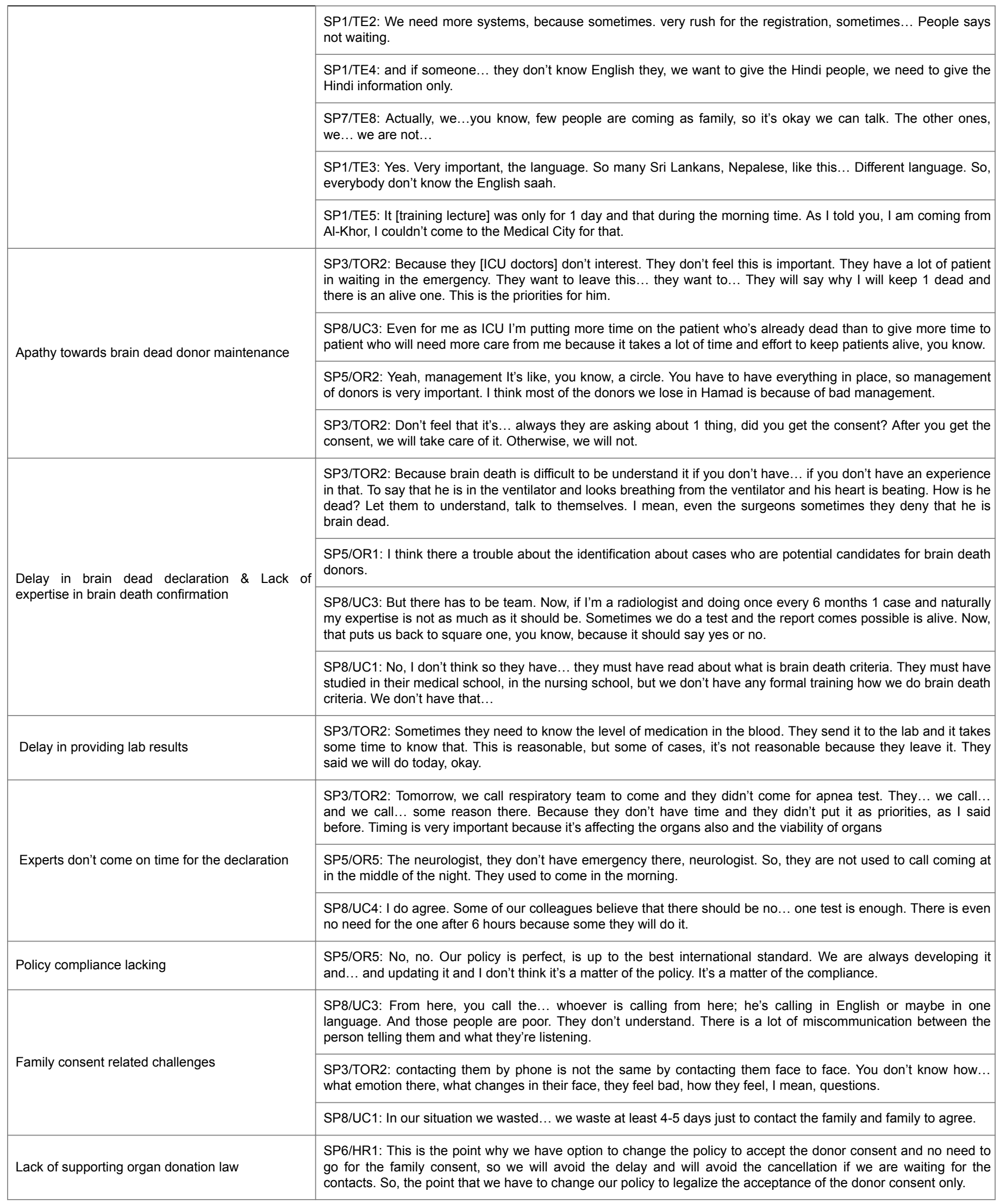


Citation: Al-Thani H, Agarwal TM, Singh R, Varughese B, Al-Maslaman Y, et al. (2018) System Level Challenges and Resolutions in Organ Donation and Transplantation Program in Qatar. J Transplant Technol Res 8: 178. doi:10.4172/2161-0991.1000178

Page 4 of 13

Under utilization

Interdepartmental communication gap

Lack of work up protocol for recipients and donors

Challenges faced by organ recipients in Department of Emergency

Lack of facility for workup of living donors in Satellite hospitals

Infrastructural challenges
SP5/OR3: And it was astonishing that there is another teams from, I think, Saudi Arabia, okay, they take... they take everything. Liver, heart, kidneys, everything. I asked why they are doing that one? We... we need those kidneys, I think. Why did you take the ears, their eyes, the kidneys? They said because we have very high standardized. This kidney is not working for us. This is as I remember. So, it's working for them and not working for you? And this is I couldn't understand it.

Participant: Ya ya, right. I remember when <Name> he sent for me the list of like a certain organs they will not accept the kidney because the patient has fever... necrocytosis. Even they are not consulting us to take the organ or no. Because if the patient has white blood cells or necrocytosis this is infectious disease matter. Right? They are not consulting us. They will reject without ID consultation. You know and we will do all the necessary work; and by the way we will hear from liver transplant but we will not hear anything from the kidney transplant.

SP8/UC1: The other organs we have to wait for the other country to come and take it, so it's a delay again. We have to contact them and they come maybe a couple of hours' time or they may come in the night when they have their free time, so that also delays to take the organs.

SP5/OR5: You are bringing it from abroad?

SP5/OR3: Usually, I give the priority for the Qataris, okay.

SP6/HR1: We don't have ophthalmologist in the transplant committee. We don't have... even I don't think that they are taking the cornea from our patients.

SP6/HR1: From both sides. I think there is a problem from both sides. We didn't communicate and they didn't show interest.

SP5/OR3: I did not receive in the last 5 years, I didn't receive anything [donated cornea] from the... this committee, for donation committee. Eyes, all abroad... outside. And we are buying the cornea. Iran. Tehran, Iran.

SP5/OR3: He should pay the cornea, patient will not discharge unless he pay. That's important. Well, I have a list of Qataris and non-Qataris. Usually, I give the priority for the Qataris, okay.

Participant: The department of infectious disease is not involved in the transplant process by the team of the kidney transplant. Though earlier the infectious disease physicians were involved during pre-transplant assessments, now they are not called even for urgent kidney transplants.

SP3/TOR1: Hmmm. There is no clear protocol said when I can start with donor. Sometimes they told us recipient when he is in waiting list, you can start with donor, okay. After that they said no. Any donors come, don't say no for him, and start with them. I still it's not clear for me as coordinator when I can start with the donor. Really, it's not clear for the coordinator.

SP3/TOR2: Before they said don't start with donor because sometimes we finish a donor and ready and the recipient, he is unfit. Okay, we you get me to work with the donor all this time 2 and 3 months, go and come, and the recipient he is unfit.

SP4/TOT1: If our consultant call the consultant of the emergency, he refusing to give the patient. What we are doing? You tell the patient go direct by yourself for emergency and tell I'm sick because there is no good pathway for our transplant case to admit through emergency or admit in hospital itself.

SP4/TOT3: Hamad, in the sense, we have different satellites for the Wakra Hospital and Al Khor. Whoever is with the kidney patients in Qatar, we are the ones who is leading the workup.

SP5/OR5: If I talk with the family and they find that there is a cannula in the patient and he's cannulated before I take the consent, there will be a big problem. So, the non-heart beating is still not here. When there is a mature donor registry, most of the people are there on the donor registry, or there is a presumed consent system which is against the law, against the system here, against religion.

SP8/UC3: It's not the... the system is not ready.

SP8/UC1: The patient comes, had a cardiac arrest, resuscitate, could not resuscitate, within half an hour, we are supposed to take the organs out. Again, as an expatriate, no relatives, who will give the consent for them?

SP8/UC3: In the expatriate population, I think we should have camps and each camp like Nepali, so people should be taught in that particular language, you know. Not in English or posters, you know, they can't even read.

SP5/OR3: And this is the supposed to contain the corneal bank but it is stopped somewhere and we didn't hear about it and this is it. So, this is... the problem is there is no coherent communication...

SP5/OR3: But I need the corneas. I had to go borrow to do the new techniques, the machines from the private Hospital. Do you believe that?

SP5/OR5: If we have... if we have at least to admit them, finding a place to admit them the case is not easy. Sometimes we have the organ ready and we don't have a place to admit the patient to do a transplant next day. 
Citation: Al-Thani H, Agarwal TM, Singh R, Varughese B, Al-Maslaman Y, et al. (2018) System Level Challenges and Resolutions in Organ Donation and Transplantation Program in Qatar. J Transplant Technol Res 8: 178. doi:10.4172/2161-0991.1000178

Page 5 of 13

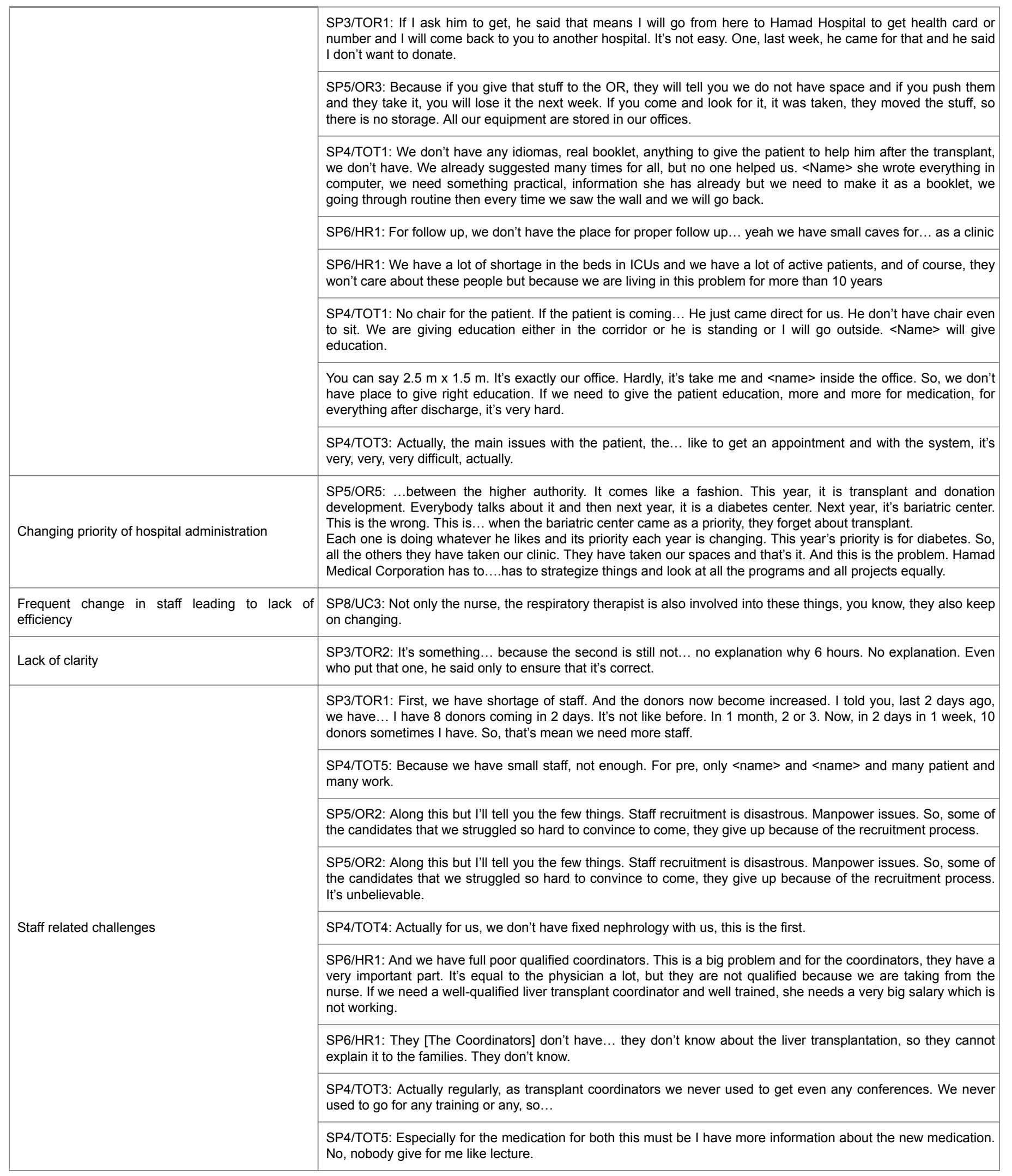


Citation: Al-Thani H, Agarwal TM, Singh R, Varughese B, Al-Maslaman Y, et al. (2018) System Level Challenges and Resolutions in Organ Donation and Transplantation Program in Qatar. J Transplant Technol Res 8: 178. doi:10.4172/2161-0991.1000178

Page 6 of 13

\begin{tabular}{|c|c|}
\hline & $\begin{array}{l}\text { SP6/HR2: But it is the ICU nurses and physician [in satellite hospitals] need to be educated.[About how and when } \\
\text { to make referrals]. }\end{array}$ \\
\hline & $\begin{array}{l}\text { SP8/UC3: Not only the nurse, the respiratory therapist is also involved into these things, you know, they also keep } \\
\text { on changing. As we have discussed, we should have one team, a brain-dead team who knows what the } \\
\text { procedure is. We don't have to keep on teaching them... }\end{array}$ \\
\hline & $\begin{array}{l}\text { SP5/OR3: Nice, you bring that one. Nice, you bring this one. This happened in the last... today, one my fellow, } \\
\text { he's supposed to do a cornea transplant, okay and we have problem that with a patient, he will do for non-Qatari } \\
\text { patient supposed to be, non-Qatari patient, okay. That patient, he has no money. I said okay, please send a letter } \\
\text { to the Medical Education, this will be a training purpose, so please let's just take that money away, for the present } \\
\text { patient. They agreed, and patient done surgery, there now processing the surgery, finance they refused. He } \\
\text { should pay the cornea, patient will not discharge unless he pays. Why? Is this a process of training? I want to train } \\
\text { my fellow. Still no policy enforced. }\end{array}$ \\
\hline & $\begin{array}{l}\text { SP5/OR5: Nursing under us which is different. The problem with our programs is more program. When we do } 4 \text { or } \\
5 \text { cases per year for me to take cases or even } 20 \text { cases, hopefully this year we will reach } 20 \text { cases which is not } \\
\text { enough to train those who have no training. Get my point? }\end{array}$ \\
\hline \multirow{2}{*}{ Policy non-compliance and need for new policies } & $\begin{array}{l}\text { SP5/OR5: No, no. Our policy is perfect, is up to the best international standard. We are always developing it } \\
\text { and... and updating it and I don't think it's a matter of the policy. It's a matter of the compliance. }\end{array}$ \\
\hline & SP6/HR2: We are planning to change the policy that all brain death, once we have the first test to do the HLA. \\
\hline
\end{tabular}

Table 2: Illustrative quotations for each them.

Results indicated that timely brain death declaration, organ procurement related delays, lack of multi-disciplinary teams while deciding about usability of available organs, lack of participation from all departments conducting organ transplant in the organ donation committee, communication gap and training related issues were the main factors influencing organ donation system in Qatar. These were ultimately leading to underutilization, wastage of available resource and low number of family consents.

\section{Organ donation campaign challenges}

Staff training: Educators at organ donation campaign often do not find their training satisfactory, as it did not equip them to answer the different kinds of questions that incumbents ask. The discussions brought out that the two main challenges impacted educators' training; firstly, it was conducted only at a single location within the country and at one fixed schedule; this made access difficult. Secondly, the training lacked practice component or inclusion of questions asked during the previous year campaign, which made campaign educators feel under equipped.
Language of coordinators: Coordinators are not able to communicate in different languages hence information about organ donation could be provided only to those who spoke their language.

Lure of incentives: Some of the campaign educators tended to give incomplete or inaccurate information so as to lure more number of people into registering as donors, which resulted in getting incentives from organ donation center. Hence, some of these registering donors were non-serious and unaware people are registering as donors. The most common confusion that is created during this transaction is assuming that the donor card is equivalent to the health card and makes them eligible to free health treatment in the hospital. Often when this gets clarified, people come back and cancel their registrations.

Communication of donation decision: During the group discussions, the campaign staff reported that they never asked the registering individual to inform his family about his donation decision (Table 3).

\section{Suggestions made by participants}

Organ donation registration

Improve impact of organ donation campaigns

\section{Illustrative remarks}

SP6/HR3: The second thing about the whole population as the citizens or even people who live in Qatar. We knew... this happened in the West. When you're going to renew your iqama here or your IDs there, so they give you a piece of paper and there's everything about, you know, donation and then if you accept already, you sign and submit, and you are now patient donor. So, something like this could be applied here for everybody plus media and orientation.

SP8/UC3: In the mall, maybe 100, 200 to 3000 people but if you have camps in particular areas for different communities, you know, then and they are given food and, you know, just a onehour lecture and some interaction with religious leaders, to start somewhere and then every 2-3 months again, keep on repeating it.

SP8/UC4: I think it will be more effective if we approach groups rather than, you know, inhomogeneous people. If there is a way of approaching communities, for example, we have our own club or own community and so on like this, arrange it. 
Citation: Al-Thani H, Agarwal TM, Singh R, Varughese B, Al-Maslaman Y, et al. (2018) System Level Challenges and Resolutions in Organ Donation and Transplantation Program in Qatar. J Transplant Technol Res 8: 178. doi:10.4172/2161-0991.1000178

Page 7 of 13

\begin{tabular}{|c|c|}
\hline & $\begin{array}{l}\text { SP1/TE5: A proper } 1 \text { or } 2 \text { hours we will have discuss what... like previous years who all } \\
\text { attended, they can write and give what all questions they have and we will try to find answer for } \\
\text { the next campaign. }\end{array}$ \\
\hline & $\begin{array}{l}\text { SP1/TE5: What I suggested that if we are a group of people from } 1 \text { particular area or hospital, } \\
\text { we can have a sometime discussion on this and there will be a team leader from that hospital, } \\
\text { they can give information rather than coming to all the way here, it would be better then, or } \\
\text { send the emails so they can come... before coming to this thing we can read that. }\end{array}$ \\
\hline & SP7/TE8: Yeah, multi-language people. If you allocate in each site then it will be well. \\
\hline \multirow[t]{4}{*}{ Allocated team for brain death declaration and maintenance } & $\begin{array}{l}\text { SP8/UC3: This was told to them that there should be a team. It's a rapid response team like } \\
\text { once we do the test, the other team comes and takes over, you know. }\end{array}$ \\
\hline & $\begin{array}{l}\text { SP8/UC4: What are the best suggestions I think, your suggestion if we wait in our roster rota a } \\
\text { brain death on-call. }\end{array}$ \\
\hline & $\begin{array}{l}\text { SP8/UC1: So, we have to finish } 6 \text { hours. We are always on-call, as a neurologist has to come. I } \\
\text { know they are busy in other set up or something like that or maybe they have... they can have } \\
\text { separate team for coming and doing the test at late night also. They can have separate team } \\
\text { and they can come, we call them and they can come at regular any time and do it... }\end{array}$ \\
\hline & $\begin{array}{l}\text { SP8/UC3: Yes, a separate response team. } \\
\text { But there has to be team. Now, if l'm a radiologist and doing once every } 6 \text { months } 1 \text { case and } \\
\text { naturally my expertise is not as much as it should be. So, if there is a team for radiologist only } \\
\text { to do their specialized people like interventional radiologist, there are many specialized } \\
\text { radiology. Sometimes we do a test and the report comes possible is alive. Now, that puts us } \\
\text { back to square one, you know, because it should say yes or no. Then it doesn't help us. So, if } \\
\text { the person who's doing every... every... every week or possibly after } 1 \text { year, he will be expert. } \\
\text { He will just document, that's it. }\end{array}$ \\
\hline \multirow[t]{3}{*}{ Change in law regarding consent } & $\begin{array}{l}\text { SP6/HR1: This is the point why we have option to change the policy to accept the donor } \\
\text { consent and no need to go for the family consent, so we will avoid the delay and will avoid the } \\
\text { cancellation if we are waiting for the contacts. So, the point that we have to change our policy to } \\
\text { legalize theacceptance of the donor consent only. }\end{array}$ \\
\hline & $\begin{array}{l}\text { SP6/HR5: I think the donor card, what the donor card says, the statement on the donor card is } \\
\text { very important. It says that I am an organ donor and I would like to donate and I have discussed } \\
\text { with my family. So, once you put this statement on the card this means that, you know, it will be } \\
\text { more easy and if the person has already discussed then you are not bound to discuss with } \\
\text { somebody who might say no. Why should I take the right away from that person? }\end{array}$ \\
\hline & $\begin{array}{l}\text { SP5/OR3: And you're asking them to take a donors. Even if the person he was donated in life } \\
\text { that, I'm ready... they will refuse. They will reject. They might kill you. Is it? They will not allow } \\
\text { you. Unless there is a rule telling me that you can take an organs from the... who already } \\
\text { donate his... during his life, allow. Like me, I already donated my organs, you can take without } \\
\text { relaying to my family, because I'm sure they will refuse. I'm sure. }\end{array}$ \\
\hline \multirow[t]{5}{*}{ Improve understanding about brain death } & $\begin{array}{l}\text { SP8/UC3: I think there is a problem in sometimes we should make a booklet. In different } \\
\text { languages. That booklet should be handed to the near ones, you know, that what is brain dead, } \\
\text { what the doctor is going to do and what we'll tell you and sometimes they think the heart is } \\
\text { working, so he's still alive, you know. So, people have this notion. You know, the word will } \\
\text { spread, so one will read that, he will go to his camp he will tell them this is what they gave me } \\
\text { and what do you think? They will discuss, you know, awareness will also increase. It's not only } \\
\text { that } 5-6 \text { people who come and visit the patient, you know, we don't have that and I told many } \\
\text { time, we should have it actually. }\end{array}$ \\
\hline & $\begin{array}{l}\text { SP3/TOR2: Let's just say the belief that is the brain death it's already understandable and the } \\
\text { people didn't know exactly what it's meaning is. Knowledge improvement. Could be a focus } \\
\text { group, we talked for the nurses, start with doctors. We talk person to person or person to } \\
\text { groups or group to groups. }\end{array}$ \\
\hline & $\begin{array}{l}\text { SP8/UC1: No, I don't think so they have... they must have read about what is brain death } \\
\text { criteria. They must have studied in their medical school, in the nursing school, but we don't } \\
\text { have any formal training how we do brain death criteria. We don't have that... }\end{array}$ \\
\hline & $\begin{array}{l}\text { SP8/UC3: All nurses, if they can attend fire safety, I don't know why shouldn't they attend } \\
\text { mandatory class for brain death criteria. They should be given certificate that you have } \\
\text { understood. Only then things will move. Otherwise, I get a patient who's brain dead after one } \\
\text { month. One month is, you know, one nurse will do it, other } 15 \text { nurses don't know anything. }\end{array}$ \\
\hline & $\begin{array}{l}\text { SP8/UC1: Per year or new students coming... coming can take the one class and yeah, } \\
\text { ultimately it belongs to the...declaration of brain dead. }\end{array}$ \\
\hline
\end{tabular}




\begin{tabular}{|c|c|}
\hline Information booklet should be provided to post-transplant patients & $\begin{array}{l}\text { SP4/TOT1: We don't have any idiomas, real booklet, anything to give the patient to help him } \\
\text { after the transplant, we need something practical, information she has already but we need to } \\
\text { make it as a booklet. }\end{array}$ \\
\hline Improving consent by appointing country representatives & $\begin{array}{l}\text { SP8/UC3: So, I would suggest that to get this thing rid off, they should have some } \\
\text { representative in Nepal or, you know, local guys, } 1 \text { or } 2 \text { who as soon as the patient is in ICU, we } \\
\text { call them because we are in direct contact. They are employed by Hamad or whichever system. } \\
\text { We call them and we go and tell the... they are going to tell the family in their respective } \\
\text { language, you know, Nepali, Bengali... because they are local guys. They can tell them that } \\
\text { your son is sick... is very sick and chances of survival less. I got a call because I'm } \\
\text { representing that country. Then there's a good rapport developed between the family and this } \\
\text { particular person. }\end{array}$ \\
\hline Increasing donor registrations & $\begin{array}{l}\text { SP6/HR3: When you're going to renew your iqama here or your IDs there, so they give you a } \\
\text { piece of paper and there's everything about, you know, about donation and then if you accept } \\
\text { already or agree, you sign and submit, and you are now patient donor. So, something like this } \\
\text { could be applied here for everybody plus media and orientation. }\end{array}$ \\
\hline Accelerating brain death testing & $\begin{array}{l}\text { SP8/UC4: if we are doing the test on the expected donor very fast and very quickly, so that we } \\
\text { can identify the most useful patients for the test. But I think if we can do this as early as } \\
\text { possible or probably once we anticipate that brain death is almost pending then I think we can } \\
\text { save sometimes in this. }\end{array}$ \\
\hline Increasing liver transplantation & SP5/OR2: One way of increasing liver transplant is having a pediatric program. \\
\hline
\end{tabular}

Table 3: Illustrative remarks for suggestions given by participants.

\section{Brain dead donor related challenges}

Brain dead declaration: Formal training on brain death criteria is not available within the hospital. Everyone deals with it based on their previous learning at nursing or medical schools.

Timing of brain dead declaration is a crucial factor in preserving the organs of a brain dead person. However, often health care workers show apathy resulting in lack of policy compliance with regard to second brain dead declaration after 6 hours of first one or providing lab results related to brain death declaration. Sometimes labs delay the test results or respiratory teams who are called for confirmatory brain death testing do not come on time.

Lack of expertise in confirmatory brain death testing due to lack of regularity in practice delays brain dead declaration as sometimes specialists give ambiguous results such as 'possibly alive' which brings back the declaration to where they started from.

Donor maintenance and management: Apathy towards care of brain dead patients when other live patients requiring ICU care are waiting for beds or refusal to maintain brain dead case unless consent has been obtained often leads to deterioration in organ viability.

Maintenance and management of the donor requires a holistic approach. Most of the donors in the hospital are lost due to poor management of donor in the duration that consent is taken.

\section{Underutilization of available organs}

Potential donors are not converted into actual donors and available organs from consented donors are not always utilized. Both these lead to underutilization and are triggered by several different system level challenges.

Lack of supporting law: Qatar organ donation law does not support organ procurement without family consent which becomes a challenge because a large number of families of potential donors who have been declared brain dead, stay in their home countries and not in Qatar.
Family consent related challenges: Language barrier and Lack of face to face communication created communication gap between the families being approached for consent and the organ donation department due to the physical distance and language barriers. Patient's family members often live in remote locations and are poorly educated hence making communication with them over phone in a language unfamiliar to them result in miscommunication and denial of consent for the donation.

Substantial time is lost in the process of making physical contact with families living in remote locations of developing countries where network connectivity is poor and in explaining, about organ donation to them during their grieving period. In many cases organ is lost during this period before consent could be obtained.

Inter departmental communication gap: Communication gap observed between the organ transplant teams and organ transplant committee. Department of ophthalmology, where corneal transplants were being carried out regularly, was not provided with information when a consented donor was available. Hence, all the corneas being transplanted in Qatar were bought from other countries. Both the members of organ donation committee as well as the participant from the department of ophthalmology believed that a big communication gap existed and both together were responsible for this situation. During the discussion it was also brought out that some of the surgeons doing transplant in Qatar were not even aware of this situation or that corneal transplants were being done in Qatar.

Absence of donation after cardiac death: Non-heart beating organ donation is not practiced in Qatar because presumed consent for organ donation is not available and any delay in organ extraction could impact the quality of the organs.

A mature donor registry is lacking in the organ donation system of Qatar. Therefore, further consent is required even if an individual had already registered his will for organ donation.

Surgeons feel under-confident about the capability of the present infrastructure in handling cases of non-heart beating organ donations. 
Organ sharing: Other countries are invited to take organs of consented donors which are not being utilized here in Qatar. This sometimes caused unwarranted delay in organ procurement.

Policy compliance: Family consent related challenges good policies have been put in place after the organ donation accord was signed. However, what really led to wastage of available organs was noncompliance. Policy related to second test for brain death after 6 hours was often not complied with.

\section{Live donation work-up related challenges}

Protocol lacking: In living organ donation there is a lack of clarity about when the coordinator needs to start work up with the donors and recipients, and in what order. Protocol is not in place and this often creates a chaotic situation putting the coordinators in a difficult position. Work-up with the living donor takes about 2-3 months; sometimes at this time, the recipient is found to be unfit and the effort of the coordinator as well as the donor goes waste.

Lack of centralized facility: This markedly affected living donation as it often became a distressing factor for donors to go from one center to another to get the different tests done to check the suitability of their organ for the recipient. Sometimes this leads them to drop out from making the donation.

\section{Infrastructural scarcity}

Campaign infrastructure: Scarcity of available registration card printers and staff to attend to incumbents in campaigns with high footfall often became dissuading factors for individuals willing to register for organ donation.

Information pamphlets and banners at campaigns were only available in English or Arabic language, which was not always well understood by all incumbents.

\section{Within hospital infrastructure:}

1. There is scarce number of beds in the ICU. Few beds are shared by several surgeons for admitting their patients. No priority was being given to transplants even when a donor becomes available especially in case of renal transplants. Sometimes, even in case of emergency, no bed was allocated and surgery was performed while keeping the patient in day care. It was difficult to find places to admit transplant patient. Though the infrastructure of the hospital was designed to cater to relatively lesser population in the year 1982, the discussion brought out that there was no increase in ICU beds or operation theaters though the population had increased multifold.

2. Facilities were not available for patients of kidney disease in the satellite hospitals. All work up was being done in the main Hamad hospital which made treatment as well as organ donation a big challenge for the receiver as well as the donor.

3. Though corneal transplants are carried out in Hamad, there was no corneal bank and state of the art surgical tools were reportedly lacking. The eye surgeons often had to borrow machines using latest technology for the surgery from private hospitals

4. Storage space was another challenge for transplant surgeons. They did not have space to keep their surgical instruments which often had to be stored in boxes in their own offices to avoid losing them from operation theaters.
5. There was a shortage of physicians in the transplant department and the rooms for follow up were extremely small in size. The room where the follow-up was done was often changed and no specific rooms were allocated for follow up of patients. Rooms of coordinators lacked adequate furniture and spaces in their room.

6. Some challenges were specifically being faced by post-transplant patients.

7. These patients had to use common waiting areas and washrooms, and never given any priority in being attended at the department of emergency, though they were on immunosuppressant and far more prone to different kinds of infections.

8. Getting referrals for post-transplant patients from physicians was considered a big challenge by the staff nurses as none was assigned to specially attend to the post-transplant patients.

9. Simplified booklet was not available for providing the patients with information about medications and side effects that they could have. Sometimes the pharmaceutical company provides complicated booklets which the patient cannot understand.

\section{Changing priorities of administration}

There is a lack of consistency in the prioritization approach of the higher administration; their priority about which illness needs more attention and more developed services keeps changing annually. There is no centralization of transplantation services in one location. Hence, people still find it difficult to find transplant facilities.

\section{Staff challenges}

Three main challenges with respect to staff in organ donation and organ transplant are related to:

Staff scarcity: There is insufficient staff in department for posttransplant patients as well as for live donation workup. Though the numbers of living donors approaching the center as well recipients who need post-transplant monitoring and care have increase multifold, the number of post-transplant coordinators has almost been constant. Recruitment of staff is another big problem faced by the teams in organ donation and organ transplant. It was reported that the process of recruitment is so cumbersome that often the selected individual gives up.

Staff training: Lack of staff affects staff training as neither can they be sent for courses out of the hospital nor the experienced staffs have time to conduct intra departmental training. Coordinators are not made to undertake any ongoing training to improve their skills, though they themselves felt need for the same, they lacked support from the department physicians. This impact overall quality of the staff. Qualification and capabilities of existing staff handling post-transplant patients was especially questioned as they lacked knowledge about the exact transplant process and medications and often failed at handling responsibilities. This results from inter department transfer of nurses to work as coordinators, rather than recruiting qualified coordinators. The importance of role of the coordinators is not well understood. Some coordinators working for getting deceased donation consents believed that more training is required in approaching grieving families for donation consent.

Since transplantation work is not carried out in the satellite hospitals of Hamad, there is a need to train the ICU staff of the satellite hospitals about when and how to make referrals. 
Medicine companies introducing new medication do not give proper training to educate the coordinators about its usage, effectiveness and side-effects. Coordinators only get to know more if complications arise and they ask the doctors. This makes coordinators feel unequipped to handle patient queries.

Intradepartmental peer training especially surgical training becomes a challenge as sometimes trainings approved by Medical education is faced with hurdles for financial clearance from department of finance. Peer training also sometimes becomes a challenge as the number of cases of transplant each year is not too many. These are not sufficient to train a totally inexperienced health care worker. Hence, the need for a well-trained staff is high in the current set up.

Staff allocation: Absence of allocated staff impacts work quality in the ICU. Physicians have to repeatedly train new staff as the nurses and respiratory therapists coming for the declaration of brain death are not fixed, they are on rotation with other wards. This greatly impacts their ability to do their work.

\section{Suggestions made by participants}

Several suggestions were made by the participants for improving potential donor and organ utilization. Firstly, there is a need to introduce stringent measures if policies are not complied to. Secondly, inclusion of experts in the field of infectious disease in the transplantation process right from the beginning to improve possibility of organ utilization. Thirdly, modify existing organ donation law so that family consent would not be required for already consented donors and improve donor registrations by introducing the option to register as an organ donor upon arrival in the country. Also, since the organ donation card that the registering individual gets already states that 'I have shared my decision with my family'; there is no further need to let any family member take away the right of an individual to donate by being asked to further consent once he is deceased. Finally, one member from each department performing transplant should be included in the organ donation committee, so as to ensure quick information communication as soon as a donor is available.

Organ donation related information and opportunity to register as a donor should be provided to all residents of Qatar while going to renew their resident ID card. If we follow this protocol which is prevalent in western countries, organ donation could be increased in Qatar.

Also, as suggested by participants, organ donation campaigns could have greater impact if they are run in communities or clubs where homogenous groups of people gather. Religious leaders or experts in the field of organ donation could educate the group about it during these campaigns. This could also be organized through embassies. Impact of campaigns could be improved by increase staff and number of card printers in campaigns with higher foot fall. Educational material should be made available in other languages. Training should include sessions from a mental health professional about how to approach the subject of making a donation of organs after death. It could also include focused group discussions or videos about some previous campaigns. Workshops should be organized in different locations for smaller groups so as to include practice sessions along with verbal instructions.

Allocation of a separate team of experts for brain death declaration and maintenance of brain dead patients would greatly improve the quality as it would improve expertise in procedures and ensure quick response. In order to ensure that policies related to brain death declaration are abided to, rota allocation needs to be made in turn ensuring timelines are met and individuals are made answerable for the delay when there is any.

Sometimes even the health care staff can be unsure about accepting brain death. Special training and repeat assessments are required to ensure that the health care workers in ICU have sound knowledge and are convinced about brain death and following organ donation process. This could positively influence identification of brain death cases that can be potential donors.

In order to improve understanding and acceptance of brain death, all nurses and health care workers involved in organ donation or transplant should undergo a one-day class to understand what brain death is. This would be very useful because, 'on the job training' is possible only for one nurse at a time and at a very low frequency depending on the number of times a brain dead patient is available for testing.

Booklet explaining what brain death is should be available in different languages for family members to read for all who are coming to visit the patient. This is important because often they see their near and dear ones after being declared brain dead with heart still beating and at times showing reflex movement of some body parts, are unable to trust the doctor. This will also help spread awareness about brain death.

Simplified booklets providing all relevant information should be provided to transplant recipients post-transplant. These booklets could have answers to post -transplant questions that come to the patient's mind including all details about medications as well as other practical details for patients. These should be printed in different languages.

Organ donation consent could markedly improve if representatives are appointed in the country of residence. Representatives could be employed in countries like Nepal and Bangladesh. These representatives could make face to face contact with the prospective donor's family, keep him updated about status of patient in ICU and build a good rapport with the family since he would be able to converse in the same language as the family. This would help build family trust before suggesting organ donation after brain death of patient. This would save a lot of time which is wasted in the consent process due to the communication gap.

Accelerating the process of brain death testing: HLA typing and cross-matching are time-consuming tests that can happen only after brain death declaration. However, if the protocols for such testing could be modified all potential brain death donors could be tested as a routine without requiring any special permission from family. It was suggested that if the same could be done it could contribute to saving time for the organ viability.

Non-compliance of policies especially in relation to brain death declaration should be handled sternly.

\section{Discussion}

System level challenges intensify the problem of organ shortage that is prevalent world over. Countries are dealing with these challenges by introducing opt out laws, streamlining the family consent process, improving effectiveness of potential donor utilization, improving education about brain death, modifying policies and protocols and 
formulating policies related to donor declaration and maintenance [5,13-16].

In the study, the core of system level challenges in Qatar lays underutilization of available organs for transplantation to suitable patients on the waitlist. This can be triggered at the various stages of the organ donation and transplantation process such as organ donor registration, maintenance of brain dead patients, organ allocation and transplantation.

In 2011 Qatar organ donation center was established [17] Since then organ campaigns in Qatar have started playing a significant role in educating people about organ donations and registering willing donors. So as to strengthen the impact of the growing list of individuals who register their consent for being donors, training of educators in delivering the right message plays a pivotal role in driving actual registrations that could lead to donation in future. One important aspect of communication which was found lacking in the current organ donation system and seldom recognized by educators was communication of registration decision to family members. This becomes especially important while approaching the deceased individual's family for donation related consent. When approached family members often experience ambiguity due to lack of knowledge about what the deceased individual must have wanted, hence tend to refuse the donation. The current family consent rate in Qatar is $20 \%$ [17]. As pointed by Abrar khan, the biggest challenge in the context of the Islamic world in obtaining family consent is that the family members lack knowledge whether the deceased family member had intentions to donate or not [18]. Prior discussion about organ donation has a positive influence on next- of- kin consent decision in opt-in systems and may play a vital role in increasing the number of actual donations [14]. This gap has been recognized and accepted by several government agencies and organizations procuring organs worldwide, some of them have started targeting communication with family as the primary objective of their organ donation campaigns [19]. Family consent plays a pivotal role in Qatar organ donation program, as the Qatari law does not allow organ donation without family consent $[11,20]$. It is time this issue should be given importance by the organ donation center that is involved in training the educators for communicating with public.

Underutilization was also being fed by communication gap that existed between the departments conducting transplants and the organ allocation committee which is responsible for informing various departments about available consented donors. This lack of communication greatly impacted the department of ophthalmology where all corneas for transplantation were being bought from other countries making unavailable to all the patients in the transplant waiting list. As per suggestions given by the participants this gap can be bridged by inclusion of a member from each of the department involved in conducting transplants within Qatar in the transplantation committee. This would ensure information is communicated to all these departments timely once a consented donor is available. Such communication gap could also be dealt-with by including a health communication specialist into the transplant process [21].

Qatar organ donation program is also faced with challenges due to non-inclusion of infectious disease (ID) physicians while assessing viability of available donor kidneys for transplant. Opinion of infectious disease physicians is crucial in managing infections that could be transmitted during transplants and assessing potential donor organ sustainability before organ procurement. It is believed that improving their access to organ procurement organization, would lead to optimal management of suspected transplant transmitted infections [22]. However, an absence of ID physicians in the renal transplant team has been leading to discard of kidneys that may have been viable but not of the best quality. This is usually done to ensure highest success in transplantation results and may be causing wastage of available organs in Qatar. Such wastage is not uncommon; different parts of the world have reported that transplant centers discard a significant number of the received usable organs which indicates the subjectivity and imperfections of the selection criteria [23-25]. It was recommended that this wastage could be minimized if an ID expert is a part of the team while evaluating the available organs for transplantation. Simpler complications such as necrocytosis for which kidneys are currently often being discarded could be resolved by infectious disease physicians and more kidneys could be made available for transplants. It has been suggested in several researches that increasing utilization of available organs from consented donors could play an important role in addressing the need of several patients who die while still being on the waitlist for organ donation [25-27].

Apathy towards care of brain dead patients and irregular policy compliance with regard to brain death testing timelines for declaration of brain death was reported during the study. Non-compliance was also reported in another research published in Qatar in 2014 [28]. It was found that the confirmatory test for brain death was completed $\geq 6$ hours in $29 \%$ of patients and in $70.8 \%$ of patients the second test for brain death exceeded the 6 hour threshold. Irregularity in compliance of this policy could be possibly affecting the management of patients as well as the time in which family of the patient is approached.

A research done in Qatar concluded that the somatic survival of brain dead patients is relatively short and is 3-4.5 days in Qatar which is comparable to several other countries [29]. However, as reported by participants of the present study, the time for getting consent from families living in remote locations is nearly 4-5 days or more at times. This makes delay in getting consent, an important factor behind the gap in potential brain dead donor utilization. Family consenting related challenges are multi-dimensional and impact the existing deceased donation program as well as act as one of the main hurdle in starting non-heart beating donation in Qatar. Organ donation awareness campaigns are being conducted throughout the year in Qatar and especially during the holy month of Ramadan where people are not only made aware about organ donation but also have the option of registering as a donor. Every year there has been marked increase in the number of registered donors. Therefore, making amendments in the existing organ donation law 15 support final will of the registered donor rather than family consent for procuring organs from a brain dead patient. It would not only help to increase utilization of potential donors and increase the impact of organ donor campaigns, but also increase possibility of starting non-heart beating donations in Qatar. According to the participants this becomes more important in Arabic culture where people are more resistant in giving family consent sometimes even if they know the individual had himself registered for the donation. Precedence has been set by states in the USA have adopted the Uniform Anatomical Gift Act (UAGA), (1968, 1987 revised in 2006) which has been codified into law. This act reinforces the "opt-in" system that honors the free choice of an individual to give first person consent for donation of his or her organs and is irrevocable and does not require concurrence of any other person after the donor's death [30].

Absence of brainstem function, deep unresponsive coma, and apnea are considered as the three cardinal features of brain death. However, 
Page 12 of 13

the practical aspects of brain death declaration have not been standardized in Qatar. Ancillary tests are always found to be necessary if certain parts of the apnea test or neurological examination cannot be performed reliably, or their validity is drawn into question. In Qatar the specialists involved in ancillary brain death testing are assigned for the same on rotation basis as a result of which they often lack the expertize to come to a positive or negative conclusion. This results in delay of brain death declaration. According to a research studying the pitfalls in brain death testing, one of the significant practical aspects that affect brain death declaration is specialty and training of the assessing physician [31]. It was recommended that standardized training in brain death declaration should be uniformly provided to all physicians involved in brain death declaration. Centralization of the organ donation facility and an allocated team for performing these tests could be the most effective solution for dealing with this challenge.

No simplified information booklet was available to explain about life post-transplant, newly introduced medications and their side effects. This increases work load of the already scarce coordinator staff in the post-transplant department as patients kept coming back to the coordinators with questions and had greater tendency to not comply with the prescribed medication regime. This was further complicated by the fact that coordinators were never trained by the pharmaceutical companies in the use of the new medications and their possible side effects, which impacts the quality of education that they are able to provide to the patients. A multi-center online survey about impact of multi-generic immunosuppressant medication on coordinators was able to bring out that increased workload on coordinators could impact patients resulting in high rate of rejection and graft loss and poor patient adherence. The same study also suggested that multiple generic immunosuppressive therapies must be evaluated by transplant centers in the context of challenges to transplant patients and transplant coordinators [32].

Infrastructural challenges seem to impact organ donation and transplantation process right from printing of donor cards to maintenance of brain dead donors, transplantation timeline and management and emergency care of transplanted patients. Lack of beds in ICU was repeatedly stressed during the discussions. The number of ICU beds is an indicator of the quality of the healthcare infrastructure in any country [1] besides it has an influence on the difficulties in detecting potential brain dead donors and maintaining them effectively until the completion of brain death diagnosis and procurement of organ [33]. According to research this has direct influence on the number of potential donors that can be converted to actual donors. A centralized facility for evaluation and care of donors and recipients could help address this challenge.

Qatar has a well-established and ethically sound program for living organ donation to family and closely related acquaintance. Living donation is the most accepted and prevalent form of organ donation in Qatar. Therefore, more organs are transplanted from living donors each year than from deceased donors. During the discussions, living donation challenges such as lack of work up order for donors and recipients and centralized facility for workup that lead to loss of donors as well as efforts of coordinators were brought out by the participants. Such challenges have to be addressed as they indirectly impact utilization of the consented donors by acting as a dissuading factor.

\section{Conclusion}

The organ donation and transplantation program in Qatar has been progressive since its inception and especially after the signing of Doha Donation Accord. Expat as well as Qatari community in Qatar has benefited radically from the altruistic provisions of Doha Model which is well placed in practice, and an example for the world to follow. This research brought out some of the main system challenges in deceased and living donation programs in Qatar which could be worked upon to create a more stream lined organ donation program. It was identified that there is a need for a centralized facility and standardized protocols for handling patients during the registration process, maintaining them as potential donors, assessing as donors and managing after transplantation. The infrastructure and ongoing training especially in relation to brain dead identification and care needs upgradation. The organ donation law needs to be strengthened so as to support registered organ donor's final will. Although, Qatar Government and its organizations are dedicated to continuously improving the system, healthcare individuals' responsibilities cannot be denied to make the system at par with best developed system in the world.

\section{Competing Interests}

The authors declare no conflict of interest in this research study.

\section{Acknowledgement}

Ethical approval (\#14227/14) of the study was given by IRB, Medical Research Center, HMC, Doha, Qatar. This research was presented at the Hamad Medical Corporation, Medical Research Center's Annual Research Day 2017 in the form of poster. We appreciate contribution of Medical Research Center, HMC for facilitating the study. We acknowledge constant support of Prof. Riadh Fadhil, Director, Qatar Organ Donation Center, HMC and also grateful for the support of the research project team Dr. Nacera Ouslimane, Ms. Saksheena Mekkodathil and Ms. Nazia Hirani.

\section{Funding}

Qatar Nation Research Fund (QNRF), Qatar under the program NPRP7-965-3-247, sponsored study. QNRF does not have any role in design and conduct of the study.

\section{Authors' Contribution}

Al-Thani H: Conceptualized and designed the study with the team, prepared the first draft of the research paper. Prepared the final draft and critically revised the content. Provided approval for the manuscript version submitted

Agarwal TM: Moderated the focus group discussion, coded the results on NVivo and analyzed the results. Assisted in the preparation of the first draft and approved the final submitted version.

Singh R: Analysis and interpretation of data, critically revised the draft and provided approval for the submission of manuscript and corresponding author for the study.

Varughese B: Interpretation of data, revised manuscript critically for content and provided final approval to the manuscript version submitted for publication.

Al-Maslamani Y: Participated in conceptualization and design of the study with the team. Contributed to the final draft and critically 
Citation: Al-Thani H, Agarwal TM, Singh R, Varughese B, Al-Maslaman Y, et al. (2018) System Level Challenges and Resolutions in Organ

Page 13 of 13

revised the content. Provided approval for the manuscript version submitted.

El-Menyar A: Participated in design of the study with the team and critically reviewed the draft content. Provided approval for the manuscript version submitted.

\section{References}

1. Shepherd L, E O'Carroll R, Ferguson E (2014) An international comparison of deceased and living organ donation/transplant rates in opt-in and opt-out systems: a panel study. BMC Medicine 12: 131.

2. George S, Thomas M, Ibrahim WH, Raza T (2016) Somatic survival and organ donation among brain-dead patients in the state of Qatar. BMC Neurology 16: 207

3. Hassanien AA, AL-Shaikh F, Vamos EP, Yadegarfar G, Majeed A, et al. (2012) Epidemiology of end-stage renal disease in the countries of the gulf cooperation council: a systematic review. JRSM Short Rep 3: 38.

4. Lim YS, Kim WR (2008) The global impact of hepatic fibrosis and endstage liver disease. Clin Liver Dis. 12: 733-746.

5. Shafer TJ, Wagner D, Chessare J, Zampiello FA, McBride V, et al. (2006) Organ donation breakthrough collaborative: increasing organ donation through system redesign. Crit Care Nurse 26: 33-42.

6. DuBois JM, Anderson EE (2006) Attitudes toward death criteria and organ donation among healthcare personnel and the general public. Prog Transplant 16: 65-73.

7. Collins TJ (2005) Organ and tissue donation: a survey of nurse's knowledge and educational needs in an adult ITU. Intensive Crit Care Nurs 21: 226-233.

8. Kim JR, Elliott D, Hyde C (2004) The influence of sociocultural factors on organ donation and transplantation in Korea: findings from key informant interviews. J Transcult Nurs. 15: 147-154.

9. Berkwits M, Inui TS (1998) Making use of qualitative research techniques. J Gen Intern Med. 13: 195-199.

10. Ajzen I (1991) The theory of planned behavior. Organizational Behavior and Human Decision Processes. 50: 179-211.

11. Tong A, Sainsbury P, Craig J (2007) Consolidated criteria for reporting qualitative research (COREQ): a 32-item checklist for interviews and focus groups. Int J Qual Health Care 2007 19: 349-357.

12. Agarwal TM, Singh R, El Menyar A, Al Thani H, Al Maslamani Y, et al. (2018) Perception of health workers about barriers impacting organ donation in qatar: A Focus Group Study. OSJ 3.

13. Wight C, Cohen B (1997) Organ shortages: maximizing the donor potential. British Medical Bulletin 53: 817-828.

14. Murray L, Miller A, Dayoub C, Wakefield C, Homewood J (2013) Communication and consent: discussion and organ donation decisions for self and family. Transplant Proc 45: 10-12.

15. Willis BH, Quigley M (2014) Opt-out organ donation: on evidence and public policy. J R Soc Med 107: 56-60.
16. Kosieradzki M, Jakubowska-Winecka A, Feliksiak M, Kawalec I, Zawilinska E, et al. (2014) Attitude of healthcare professionals: A major limiting factor in organ donation from brain-dead donors.

17. Almaslamani Y, Almalki H, Fadhil R, Khalaf H, Asim M (2016) Transplantation in Qatar. Transplantation 100: 2487-2488.

18. Khan A (2009) Middle east health magazine: brain death legislation and organ transplantation in the Islamic world.

19. Morgan SE, Miller JK (2009) Beyond the organ donor card: The effect of knowledge, attitudes, and values on willingness to communicate about organ donation to family members. J Health Commun 14.

20. Hamad Medical Corporation (2015) Hamad Medical Corporation: Law No. (15) of 2015 on regulating the human organs transfer and transplantation.

21. Etheredge HR, Penn C, Watermeyer J (2017) Interprofessional communication in organ transplantation in Gauteng province South Africa. S Afr Med J 107: 617-620.

22. Burdette SD, Miller RA, Levi M, Beekmann SE, Polgreen P, et al. (2012) Identifying communication gaps for solid organ transplant infection transmission among infectious disease physicians: an emerging Infections network survey. Transpl Infect 15: 8-13.

23. Morris JA Jr, Wilcox TR, Noreuil T, Frist WH (1990) Organ donation: a university hospital experience. South Med J 83: 884-888.

24. Yasinsk E (2016) When donated organs go to waste. The Atlantic Health.

25. Ojo AO, Heinrichs D, Emond JC, McGowan JJ, Guidinger MK, et al. (2004) Organ donation and utilization in the USA. Am J Transplant 9: 27-37.

26. Saidi RF, Hejazii Kenari SK (2014) Challenges of organ shortage for transplantation: solutions and opportunities. Int J Organ Transplant Med 5: 87-96.

27. http://www.bendbulletin.com/health/organtransplants/1375026-151/ transplantable-organs-go-to-waste.

28. Al-Maslamani Y, Muhsin ASA, Ali OIM, Fadhil RA, Abu Jeish AHR (2014) Potential brain death organ donors - challenges and prospects: A single center retrospective review. Saudi J Kidney Dis Transpl 25: 589-596.

29. George S, Thomas M, Ibrahim WH, Abdussalam A, Chandra P, Ali HS, Raza T (2016). Somatic survival and organ donation among brain-dead patients in the state of Qatar. BMC Neurology. 16(1):207.

30. Anatomical Gift Act (2006) Summary. National Conference of Commissioners on uniform state laws.

31. Busl KM, Greer DM (2009) Pitfalls in the diagnosis of brain death. Neurocrit Care 11: 276-287.

32. Parker K, Zagadailov EA, Bruno S, Wiland AM (2013) Transplant coordinators' perceived impact of availability of multiple generic immunosuppression therapies on patients, workload, and post-transplant maintenance therapy. J Trans 1-7.

33. Matesanz R (2004) Factors that influence the development of an organ donation program. Transplant Proc 36: 739-741. 